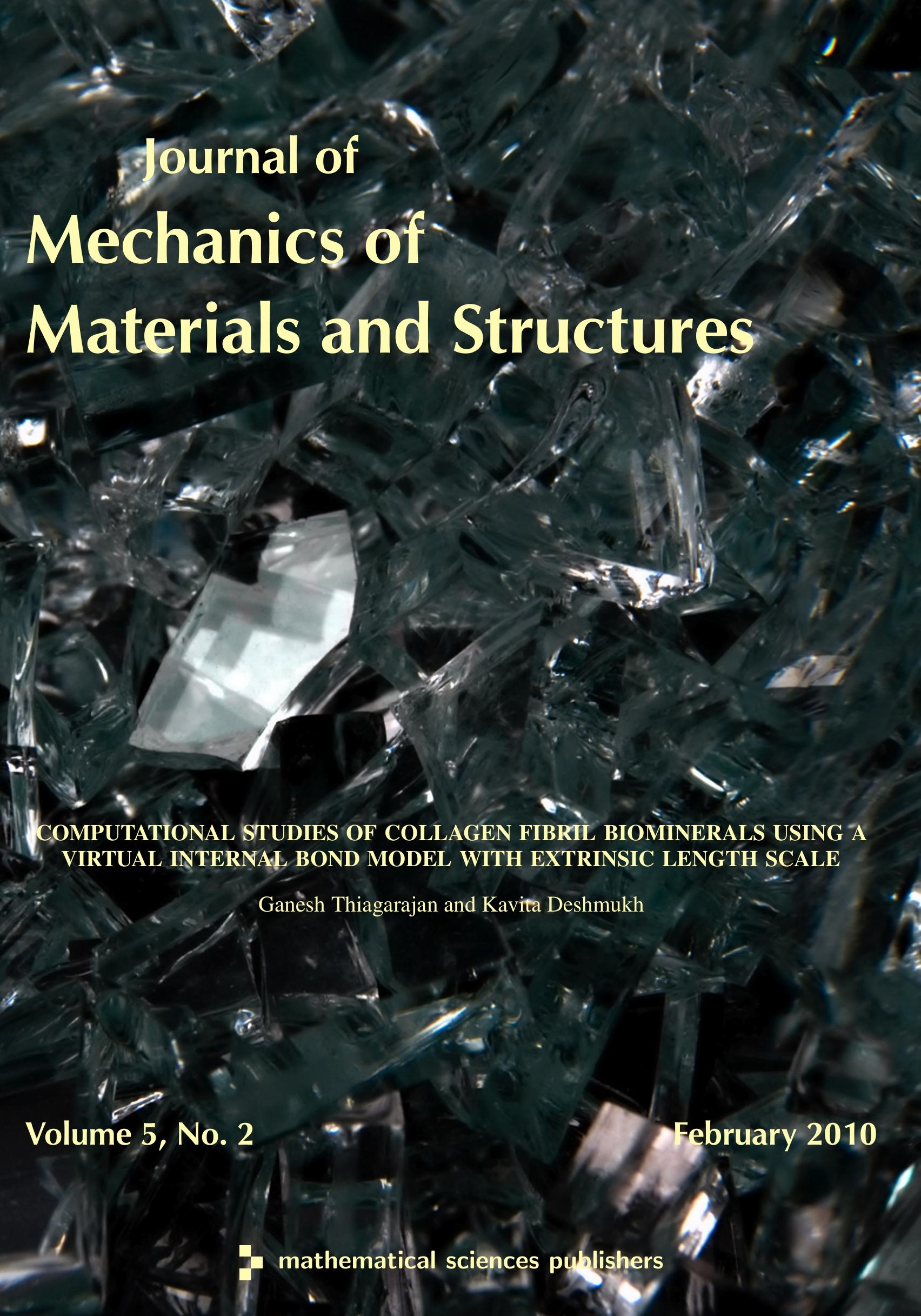




\title{
COMPUTATIONAL STUDIES OF COLLAGEN FIBRIL BIOMINERALS USING A VIRTUAL INTERNAL BOND MODEL WITH EXTRINSIC LENGTH SCALE
}

\author{
GANesh Thiagarajan AND KaVita DeshmuKh
}

\begin{abstract}
Mineralized type-I collagen fibrils are made up of mineral hydroxyapatite and type-I collagen and are known to have good mechanical properties. Hydroxyapatite by itself is stiffer and collagen is relatively weaker. The development of a multiscale virtual internal bond model (VIB) used to model the material behavior and failure of such biocomposites was described in an earlier paper by the authors. An explicit finite element based framework using a two parameter fracture-constitutive VIB model, with an extrinsic length scale, was used in this study.

The model used in this study is a nano sized dahlite mineral crystal commonly found in collagen fibrils. Two important computational implementation characteristics are presented here; namely the effect of a material parameter used in the VIB model and the effect of thickness of the material at the nanoscale on the failure behavior. The effect of the thickness is studied in order to demonstrate the extrinsic length scale capability of the VIB model at nano length scales.
\end{abstract}

\section{Introduction}

Recent research in biomedical engineering has shown evidence that the growth and development of biological tissues is also related to the mechanical loadings on and history of the tissue. Many types of biological materials, such as bone, exhibit a hierarchical structure consisting of several levels [Katz 1976; 1980; Weiner and Wagner 1998]. The mineralized collagen fibril is one of the fundamental constructs of bone, dentin, and mineralized tendons. There are three major components of a mineralized collagen fibril; the collagen molecule, minerals, and water [Weiner and Wagner 1998]. The proportion of the three components varies for different types of tissues. The collagen molecule, which is the major protein component in the fibrils, is predominantly made of type-I collagen. The triple helical structure of the collagen molecule has an average diameter of $1.23 \mathrm{~nm}$ and is about $300 \mathrm{~nm}$ long [Weiner and Traub 1992]. The mineral component of the mineralized collagen fibril in bone consists of plate-shaped crystals of carbonated apatite [Weiner and Traub 1992]. The apatite crystals are also known as dahlite and are known to be $10 \mathrm{~nm}$ wide and $0.61 \mathrm{~nm}$ thick [Landis et al. 1996]. The diameter of the mineralized collagenous fibril, at the next hierarchical level, is of the order of 80-100 nm.

The physical structure of mineralized collagenous fibril is complex and many forms have been proposed. Apatite crystals are arranged in parallel arrays in a collagenous framework to form mineralized collagen fibrils. Orgel et al. [2000; 2001] confirmed that collagen molecules show a quasihexagonal packing in the lateral plane and conform to the Hodge-Petruska quarter stagger model [Petruska and

Keywords: finite element analysis, collagen, biomineralization, nanomechanical behavior, dentin, parametric studies.

Thiagarajan thanks the National Center for Supercomputing Applications (NCSA) for computational resources and was partially funded by the National Science Foundation Grant CMS 748085. 
Hodge 1964] in the axial plane, with a periodicity of $67 \mathrm{~nm}$ (the D period). The length of the molecule is $4.46 \mathrm{D}(300 \mathrm{~nm})$ and there are gap regions of $0.54 \mathrm{D}$. Each collagen molecule is divided into 4 segments of length $\mathrm{D}$ and a fifth segment of length $0.46 \mathrm{D}$. The $67 \mathrm{~nm}$ periodicity of the collagen molecule is made up of a $40 \mathrm{~nm}$ hole region and a $27 \mathrm{~nm}$ overlap region [Weiner and Traub 1992].

Significant advances in the development of experimental capabilities in recent years have made possible the study of biological tissues at the micro and nano scales. These advances provide the motivation for developing models at these scales and provide a mechanism to validate the models experimentally.

For mineral platelets, the implementation aspects of the scaled finite element (FE) framework in combination with the virtual internal bond (VIB) model have been demonstrated in [Thiagarajan et al. 2007]. The paper outlined a new scaled FE scheme, which facilitated study of the mechanical behavior of mineralized collagen fibrils and their constituents. A two parameter VIB model, set up in an explicit FE environment using ABAQUS, was used in the study. The effect of ABAQUS/Explicit specific parameters, when applied to quasistatic loading, has been studied and presented. The factors include the mass scaling factor (MSF) and scaled time specification (ST) in a scaled FE framework. A good understanding of how the two parameters (MSF and ST) affect the analysis has provided input into the selection of the parameters for further studies. The quasistatic nature of the explicit FE simulations was also studied.

The model used in the study was a nano sized dahlite mineral crystal which is commonly found in collagen fibrils. Two important implementation characteristics were explored: first, the usage of scaled dimensions and material properties at the micro and nano length scales and, second, the scaling of loading time without the loading becoming dynamic in nature. Using energy studies it was shown that the simulations remain quasistatic in nature.

1.1. Minerals in collagen fibrils. Mineralization processes and the arrangement of minerals in collagen fibrils and fibers are areas of extensive ongoing research [Tong et al. 2003; Chang et al. 2005; Veis 2005]. Eppell et al. [2001] performed atomic force microscopy studies on mature bovine cortical bone mineralites and report that the minerals are plate-like in shape with thickness ranging from 12 to $115 \mathrm{~nm}$ (mean $37 \mathrm{~nm}$ ), width ranging from 27 to $172 \mathrm{~nm}$ (mean $64 \mathrm{~nm}$ ), and length ranging from 43 to $226 \mathrm{~nm}$ (mean $90 \mathrm{~nm}$ ). They also point out that the large mineralites could be located at the outer surface of the fibrils and $98 \%$ of the minerals are less than $2 \mathrm{~nm}$ thick with mean dimensions of $12 \mathrm{~nm} \times 10 \mathrm{~nm} \times 0.61 \mathrm{~nm}$. Kinney et al. [2003] used a small angle x-ray scattering technique and suggested that the thickness of the mineralite crystals in human teeth, in general, is approximately $5 \mathrm{~nm}$.

1.2. Observed mechanical relations at the mineral level. In addition to the crystallite dimensions two more important factors influence the mechanical behavior at the fibril level. They are the mineral density in the fibril and the arrangement of mineral particles in the collagen matrix of the fibril [Rho et al. 1998; Weiner and Wagner 1998]. Currey [1990] suggested that as mineralization increases the elastic modulus increases. Mineral crystals in collagen have the effect of increasing the stiffness and fracture stress but reducing the fracture strain [Tong et al. 2003]. For example, the lack of the intrafibrillar minerals results in reduced elastic modulus and hardness of dentin [Kinney et al. 2003; Balooch et al. 2008].

Mineralization geometry models: Mineralization is known to initiate primarily in the gap regions. Hence, the geometric order of the gaps in the lateral direction is important in determining the mineralization pattern and geometry. Two hypotheses of the manner in which the channels are formed are concentric 
channel arrangement [Hulmes et al. 1995; Perumal et al. 2008] and a three dimensional packing model that arranges the gaps as lateral channels or holes that are one molecule thick $(\sim 15 \AA)$ and extend an unknown distance in the transverse direction [Weiner and Traub 1992]. Tong et al. [2003] have suggested that the channels run for a distance of at least $10 \mathrm{~nm}$ in the transverse direction. Hulmes et al. [1995] have discussed radial packing and order arrangements in collagen fibril and had proposed that the gaps in the collagen fibril run as concentric channels around a central fibril core. Jager and Fratzl [2000] have discussed three possible arrangements of minerals in the fibril, namely, crystals occupying only the gap zones, crystals growing into the overlap zone, and longer crystal sizes $(\sim 100 \mathrm{~nm})$ extending beyond the overlap zone and having a staggered arrangement.

1.3. Objectives of the study. This paper presents the details of the next steps in the scaled FE study of mineral platelets, namely the effect of various VIB material model parameters and the effect of the mineral geometry. The VIB model, in its present stage, has two parameters $-A$ and $B$. Parameter $A$ is related to the elastic modulus of the material and has a constant value since the material itself is not being changed. The effect of varying the parameter $B$ on the stresses and failure behavior is studied and presented in this paper. Secondly, the effect of geometry on the mineral crystal behavior is studied by varying the thickness of the mineral platelet and performing scaled FE studies.

The VIB model does not have an intrinsic length scale and consequently an extrinsic length scale must be adopted in order to represent the fracture energy of the material. The extrinsic length scale effect, when the VIB model is implemented in this scaled FE framework, is demonstrated using the thickness studies presented in this paper. The output variables studied are the notch tip stress and the strain values in the loading direction. Failure patterns have also been observed and presented for various combinations of the parameters.

\section{Materials and methods}

Biocomposites, specifically type-I collagen fibrils, have good mechanical properties. Hence, the overall objective of the research was to develop a new FE based framework by which the mechanical behavior of mineralized collagen fibrils could be studied. Firstly, the nature and issues pertaining to the modeling of nano sized mineral platelets must be addressed followed by the mechanical modeling of tissues that contain only type-I collagen such as the rat tail tendon, the structural model of which is described in [Orgel et al. 2006]; the next step would be to develop models for a collagen fibril, and finally the collagen fibril model would be statistically combined to develop the FE framework for collagen fibers. This paper addresses the implementation issues related to the modeling of a single platelet.

2.1. Virtual internal bond (VIB) model basics. The VIB model is a multiscale model that combines the behavior at the atomistic and the continuum length scales. In the VIB model [Thiagarajan et al. 2004a; 2004b], the continuum is considered as a spatial distribution of material points at the continuum level, as shown in Figure 1. The material points are represented by the integration points in a FE scheme. Each individual material point is hypothesized to represent collectively the behavior of underlying atoms. At the atomistic length scale the interaction between the atoms is represented by cohesive bonds described by a two body pair potential (see Figure 1), which results in a cohesive type interaction between material points at the continuum level. 

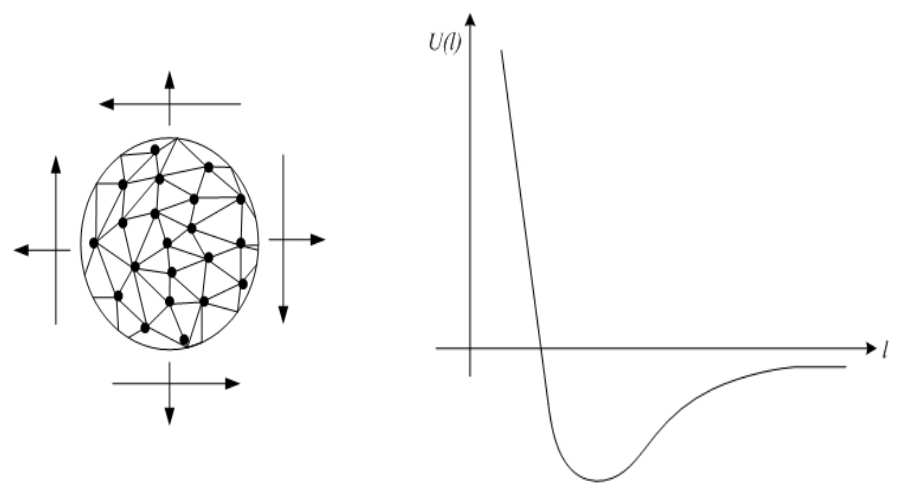

Figure 1. Atomic representation of a material point (left) and potential energy function (right).

The Cauchy-Born rule of crystal elasticity relates the motion of atoms to the continuum deformation and its measures. This rule assumes that the atomic motion, from the undeformed to the deformed configuration, under a homogeneous deformation, can be defined as a unique mapping. At the continuum level, the deformation gradient $\boldsymbol{F}$ defines such a mapping. The interaction at the atomistic level may be described through interaction potentials. In a polycrystalline material, the material behavior is isotropic due to the random orientation of grains. Such behavior can be modeled by a random spatial distribution of cohesive bonds using the VIB model. While biocomposites are a complex material, in this study it is assumed that they can be modeled as material points interconnected by cohesive bonds. The details of the original implementation of the VIB model can be found in [Thiagarajan et al. 2004a; 2004b].

2.2. VIB model framework and its suitability. The VIB model is described within the framework of hyperelastic continuum mechanics. The initial and the deformed configurations are defined using the Lagrangian coordinates $X=X_{I}$ and the Eulerian coordinates $x=x(X, t)=x_{i}\left(X_{I}, t\right)$, respectively. In this paper, capital letter subscripts are used for the initial configuration while lower case subscripts are used for the deformed configuration. In the Lagrangian description the deformation gradient can be expressed as follows:

$$
F=\frac{\partial x}{\partial X} \quad \text { or } \quad F_{i I}=\frac{\partial x_{i}}{\partial X_{I}},
$$

and the Green-Lagrange strain tensor may then be defined as $E=\frac{1}{2}\left(F^{T} F-I\right)$, where $I$ is the secondorder identity tensor.

The atomistic bond can be described by a potential energy function $U(l)$ where $l$ is the bond length (see Figure 1). Consider an arbitrary microstructural bond at angles $\theta$ and $\phi$, where $\theta$ is the angle of the bond orientation with respect to the vertical positive 2-axis, and $\phi$ is the angle in the horizontal plane with respect to the positive 1-axis. The unit vector along this direction is given as

$$
\xi=(\sin \theta \cos \phi, \sin \theta \sin \phi, \cos \theta)
$$

with respect to the undeformed configuration. The stretch of this bond can be related to the continuous deformation as $l=l_{o} \sqrt{1+2 \xi_{I} E_{I J} \xi_{J}}$. The macroscopic strain energy density function is derived using 
the Cauchy-Born rule as follows, thus linking the two scales:

$$
\Phi\left(E_{I J}\right)=\langle U(l)\rangle,
$$

where, \langle\rangle represents the weighted average with respect to the bond density function $D_{d}$ and $U(l)$ is the bond potential energy function. Assuming that all bonds have the same initial length $l_{o}$, the weighted average is given as

$$
\langle\cdots\rangle=\int_{0}^{2 \pi} \int_{0}^{\pi} \cdots D_{d}(\theta, \phi) \sin \theta d \theta d \phi
$$

The term $D_{d}(\theta, \phi) \sin \theta d \theta d \phi$ represents the number of bonds per unit volume between the bond angles $(\theta, \theta+d \theta)$ and $(\phi, \phi+d \phi)$.

2.3. Constitutive equations and cohesive force law. From the strain energy density function $\Phi$ given in (2), the symmetric second Piola-Kirchhoff stress $S_{I J}$ and the elastic modulus $C_{I J K L}$ can be derived as follows:

$$
S=\frac{\partial \Phi}{\partial E} \quad \text { or } \quad S_{I J}=\frac{\partial \Phi}{\partial E_{I J}}, \quad C_{I J K L}=\frac{\partial^{2} \Phi}{\partial E_{I J} \partial E_{K L}} .
$$

The modulus derived from this potential satisfies the major and minor symmetries,

$$
C_{I J K L}=C_{J I K L}=C_{I J L K}=C_{K L I J},
$$

as well as the Cauchy symmetry, $C_{I J K L}=C_{I K J L}$. For isotropic material this results in only one independent elastic constant due to the fact that the Cauchy symmetry is satisfied by the fourth order isotropic elasticity tensor only for the case of $\lambda=\mu$, where $\lambda$ and $\mu$ are the two Lamé's constants. Consequently, the VIB model as used in this work represents a Poisson's ratio of 0.25. The Poisson's ratio of the VIB model meshes well with the analytical modeling effort by Jager and Fratzl [2000] in their development of a mechanical model for mineralized collagen fibrils, wherein they also replaced the anisotropic nonlinear collagen by an effective elastic material with a Poisson's ratio of 0.25 , which was also used by Akiva et al. [1998] in their modeling effort.

In order to represent the potential $U(l)$, in this paper the two parameter cohesive force law, as shown in (5), is used:

$$
U^{\prime}(l)=A\left(l-l_{o}\right) e^{-\left(l-l_{o}\right) / B} .
$$

$U^{\prime}(l)$ is the cohesive force and is the derivative of the bond potential energy with respect to the bond length $l$. The constant $A$ can be related to the material shear modulus by (5) and represents the initial slope of the curve. The parameter $B$ is related to the strain at which the cohesive stress is reached.

2.4. VIB and scaled-FE modeling. Modeling the behavior of nano structures using traditional finite elements necessitates the usage of material models that appropriately represent the behavior at the nanoscale. Hence, traditional elasticity, plasticity, fracture, and damage based constitutive models may not be suitable. There are a number of requirements. For example, it has been hypothesized [Gao and Ji 2003; Gao et al. 2003] that failure of nanostructural materials is governed by critical surface energy and not necessarily by stress concentrations at crack tips. It is difficult for FE material models to incorporate this feature. In order to address these issues a new framework of a scaled FE modeling effort was presented in [Thiagarajan et al. 2007] by using the scaled material property and dimension (property scaling and dimension scaling) values at the different length scales. Another advantage of dimension scaling is that in simulations using ABAQUS/Explicit, the solution results in reduced clock times for the simulation. It was shown that as length scales decrease the simulation times also decreases. 
2.5. Geometric and VIB model parametric study. To further characterize the mechanical behavior of mineralites, scaled FE studies on specific sized mineral platelets are performed using the VIB material model. A parametric study is conducted using a notched rectangular plate model subjected to uniaxial tensile displacement based loading. The parameters studied were the VIB model parameter $B$ and the thickness of the mineral platelet $t$.

Parametric variation: The basic model studied is shown in Figure 2 which shows a mineral crystal size of $50 \mathrm{~nm} \times 25 \mathrm{~nm} \times t \mathrm{~nm}$. The dimensions chosen for this study are based on the values published in [Weiner and Wagner 1998]. Dahlite crystals can be represented as rectangular plate shaped materials with a length of $50 \mathrm{~nm}$ and a width of $25 \mathrm{~nm}$. Transmission electron microscopy and small angle $\mathrm{x}-$ ray scattering of these minerals have demonstrated a fairly uniform thickness for these minerals. The thickness varied from $1.5 \mathrm{~nm}$ in mineralized tendons to $4 \mathrm{~nm}$ for some mature bone types. Although no known measurements exist for the elastic modulus of these minerals extracted from bone, the measured elastic modulus of synthetic powdered carbonated apatite is reported in the same reference as $109 \mathrm{GPa}$.

Variation of thickness: It was shown in [Thiagarajan et al. 2007] that for a mineral platelet with an elastic modulus of $100 \mathrm{GPa}$ the value of the critical thickness $h^{*}$ comes to about $30 \mathrm{~nm}$. The critical thickness has been defined as the thickness below which flaws in the material are hypothesized to not affect the failure behavior. However, in this study of dahlite mineral, the crystal thickness of $30 \mathrm{~nm}$ is very high and hence is not considered. The values reported in [Weiner and Wagner 1998] have been used as a reference and thus thickness values of $1 \mathrm{~nm}, 4 \mathrm{~nm}$, and $10 \mathrm{~nm}$ have been studied.

2.5.1. Variation of VIB model parameter $B$. The cohesive force law, which represents the interaction between two atoms in a structure, given by Equation (5) for $U^{\prime}(l)$, has two parameters $A$ and $B$. The parameter $A$ represents the initial slope of the curve and is directly related to the elastic modulus of the material. The parameter $B$ represents the strain at which the cohesive stress (the maximum stress) is reached. A higher value of $B$ gives a higher cohesive stress value as shown in Figure 3 . A higher value

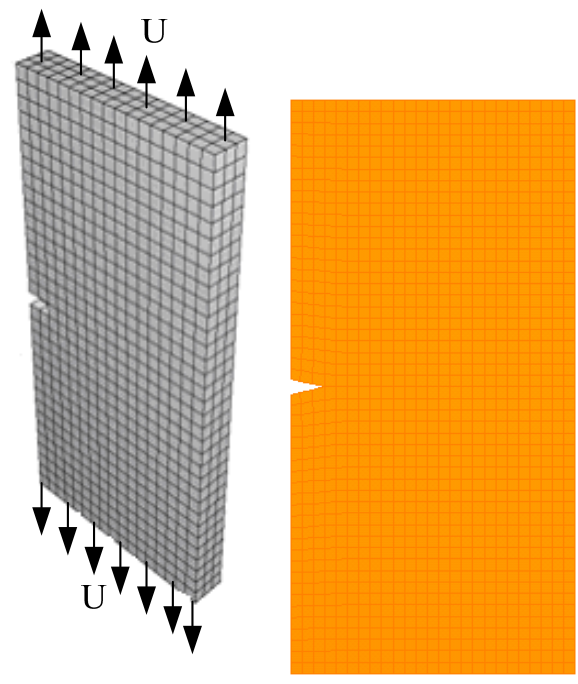

Figure 2. The basic model of mineral crystal with loading shown. 
of cohesive stress results in a greater surface energy required for the separation of surfaces in a body. Hence, from the perspective of the failure at the continuum level, it can be inferred that a higher value of $B$ represents a greater surface energy required for separation of two surfaces. Since there are no known experimental measurements of the surface energy $\gamma\left(\mathrm{N} / \mathrm{m}^{2}\right)$ for dahlite crystals, a parametric study has been conducted here with different values of $B$.

As outlined in [Thiagarajan et al. 2007], the value of the parameter $B$, using a $\gamma$ value of $1 \mathrm{~N} / \mathrm{m}^{2}$, for a $4 \mathrm{~nm}$ thick plate was 0.055 . However, due to the uncertainties in the determination of the surface energy and the theoretical failure stress values, the $B$ value is clearly not a deterministic one. Hence, the parametric study conducted here used four different values of B namely, 0.0005, 0.005, 0.05, and 0.1. Figure 3 shows the cohesive force curves for the dahlite material properties:

$$
\left(A=\frac{4 G}{\pi D_{o}^{2} l_{o}}=\frac{4 \times 100,000 \mathrm{sPa}}{\pi} \approx 127,000 \mathrm{sPa}\right),
$$

where sPa is a scaled Pascal unit, with different $B$ values. It can be seen that the cohesive stress values, given by $\sigma_{\max }=A B / e$, are equal to $23.6,236,2336$, and $4672 \mathrm{sPa}$ for $B$ values of $0.0005,0.005,0.05$, and 0.1 respectively. In Figure 3 the curve representing a $B$ value of 0.0005 is seen as a flat line due to the difference in the order of stress values represented by the $y$-axis. The cohesive stress values of the crack tip element are presented in a later section. A total of 12 different cases are thus studied, and presented in this paper, using three different thickness and four different $B$ values.

2.5.2. Loading, material properties, and other issues. The loading applied in all the twelve simulations in the study presented in this section is a displacement based uniaxial loading in the vertical direction. A specified displacement is given at both ends of the mineral as shown in Figure 2.

The time scaling (ST) concept is used in all the simulations. It was shown in [Deshmukh 2006] that a displacement application time of $1.2 e^{-1}$ seconds for the millimeter size model was equivalent to a displacement application time of $1.2 e^{-7}$ seconds for the nanometer size model. The scaled time concept

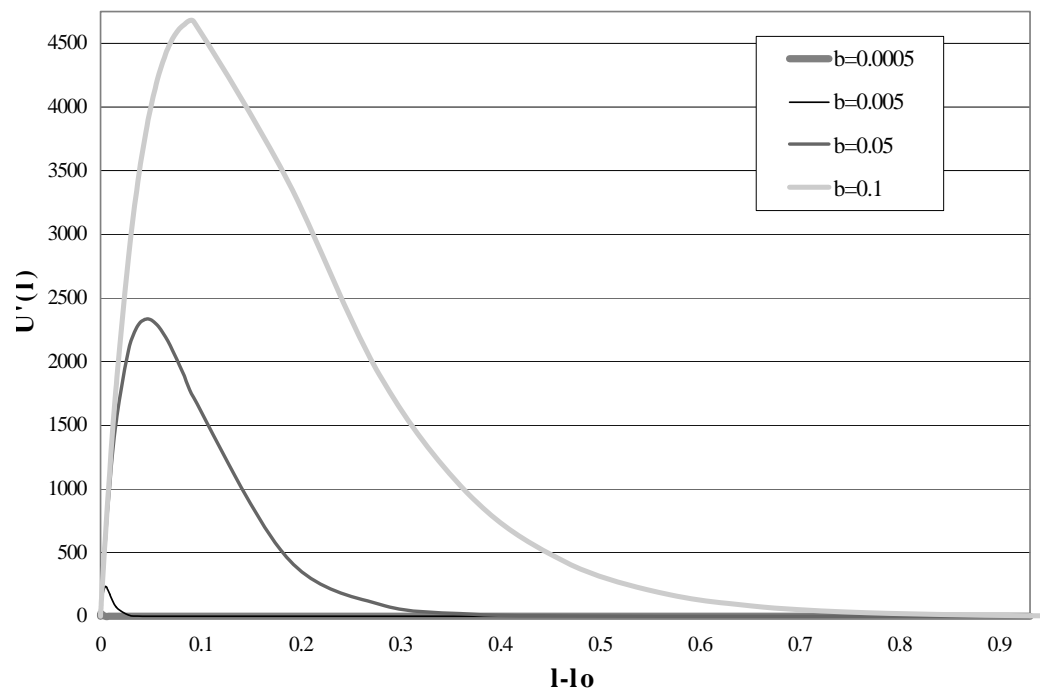

Figure 3. Cohesive force law with different values of $B$. 
demonstrated that the problem does not become a dynamic one as a result of loading applied in a very short time. As the geometric size of the model is in the nanometer range, the time of load application in all these studies is $1.2 e^{-7}$ seconds.

The MSF, an ABAQUS/Explicit based parameter, is not a variable in these studies. Based on the results outlined in [Deshmukh 2006] the value chosen here is 10,000. The values of $A=4 G / \pi$ and the density of the mineral are $50.9 \times 10^{3} \mathrm{sPa}$ and $3.18 \times 10^{-21} \mathrm{skg} / \mathrm{nm}^{3}(\mathrm{skg}=$ scaled kilogram), respectively.

\section{Results and discussions}

This section presents a comparison of the stress and strain values at the notch tip for the twelve different case studies. Stress and strain contours and graphical plots of the variation of stress and strain in the element at the tip of the notch are presented.

Notch tip stress variation: The variation of the vertical (axial) stress $S_{22}$ with time for different values of the material parameter $B$ are presented in Figures 4-7. Each graph shows the variation of the stress for the three thickness values for a given value of $B$. From the graphs, the following observations can be made:

- For $B$ values of $0.1,0.05$, and 0.005 , as the thickness increases the failure stress values increases.

- However, for $B=0.0005$, all the three thicknesses fail at the same time and with the same peak stress value. All three thicknesses fail at $1.0 e^{-9}$ seconds with a stress of approximately $64 \mathrm{MPa}$.

Failure pattern: The failure patterns of the basic model were studied for the thicknesses $1 \mathrm{~nm}, 4 \mathrm{~nm}$, and $10 \mathrm{~nm}$ for the four different $B$ values. The failure patterns for the models have been shown by depicting the axial strain contours at one time step before a total collapse was observed. Figures 8-11 show the axial strain contour and deformed shapes for $B$ values of $0.0005,0.005,0.05$, and 0.1 , respectively. The actual time step of depiction in each case is different.

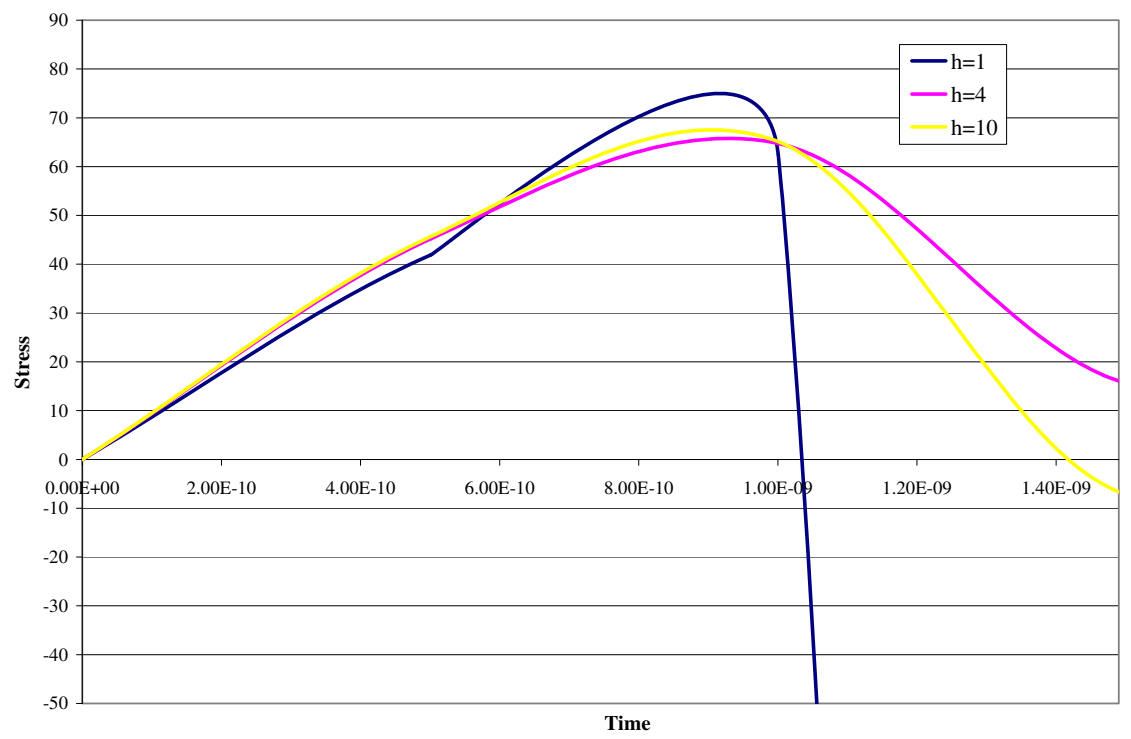

Figure 4. Variation of $S_{22}$ with time for $B=0.0005$. 


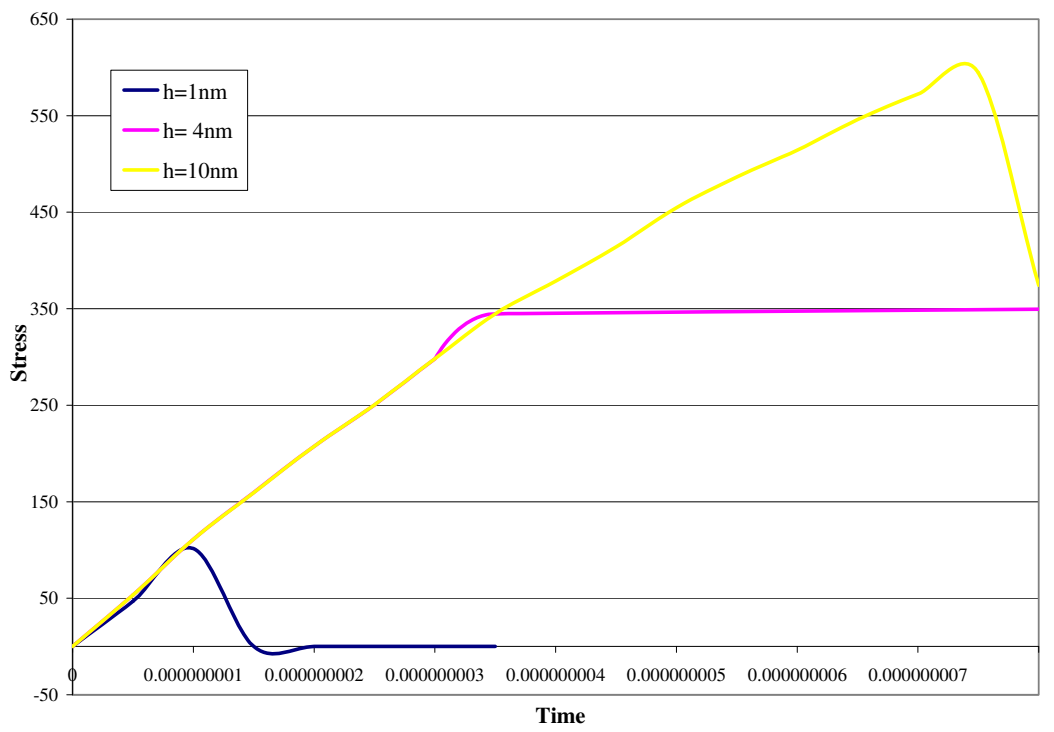

Figure 5. Variation of $S_{22}$ with time for $B=0.005$.

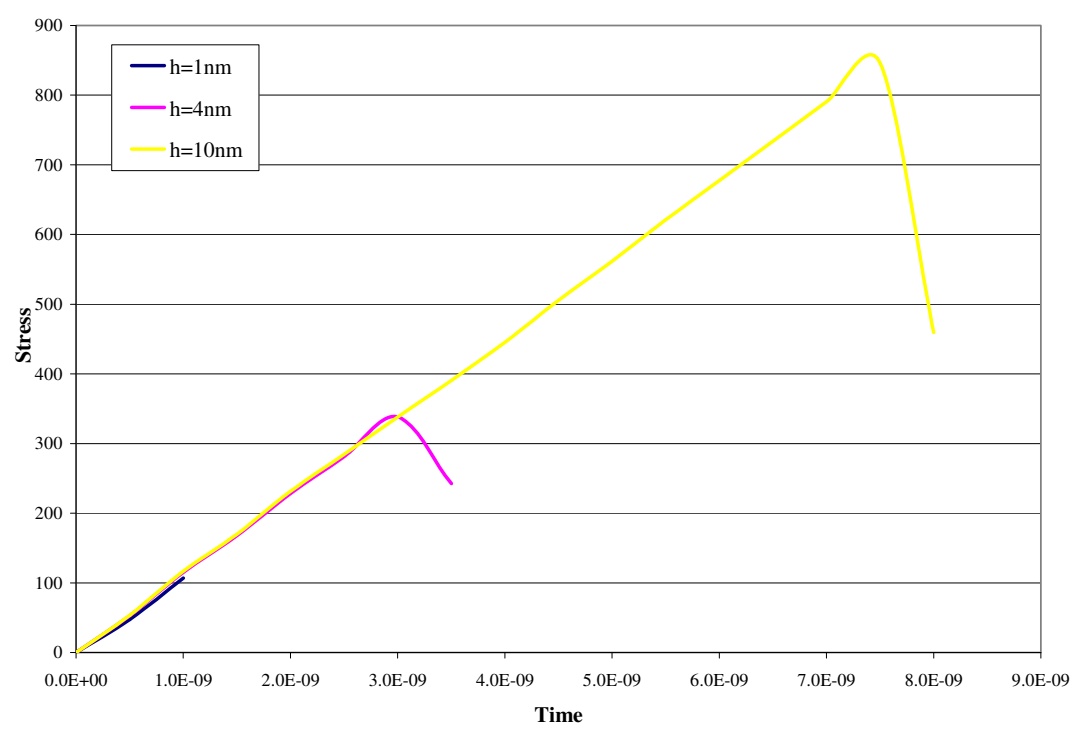

Figure 6. Variation of $S_{22}$ with time for $B=0.05$.

Failure types: There are two types of failure that can be observed from the contour plots shown. The first type is referred to as a general failure and the second type is termed the stress concentration failure.

- A general failure is defined as occurring when the whole model tends to collapse. It is proposed here that this type of failure occurs when the stresses in a majority of the elements reach close to the peak or the cohesive stress values at the same time, resulting in a subsequent loss of stiffness in almost all the elements. 


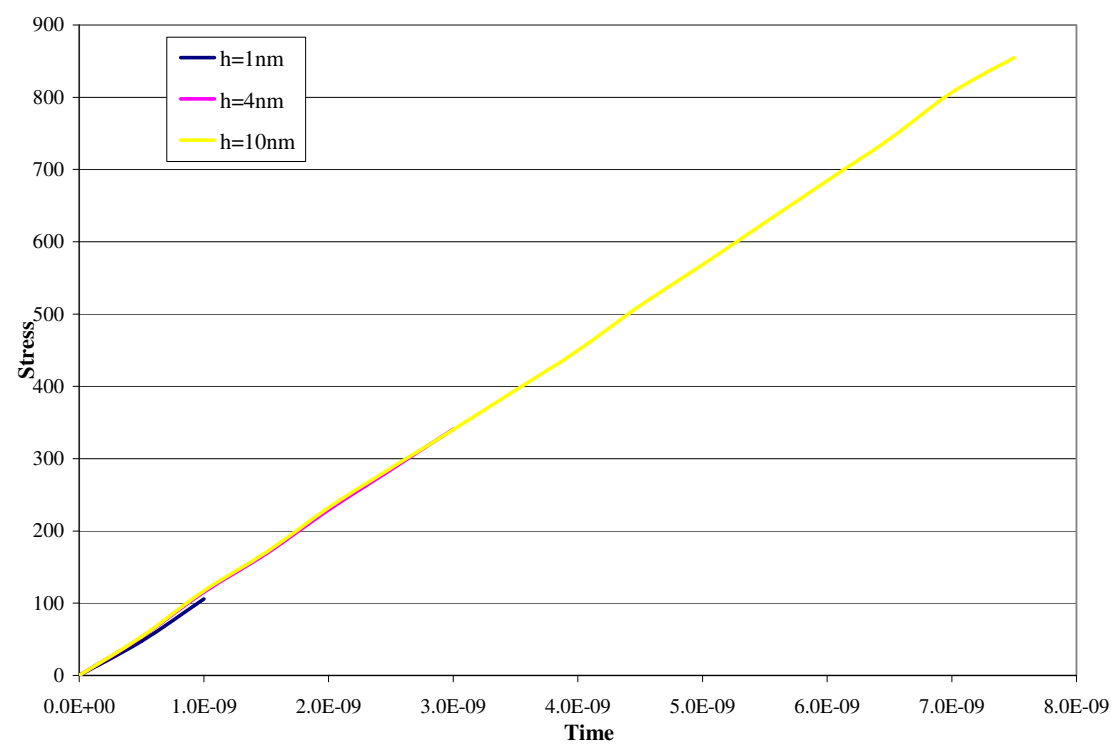

Figure 7. Variation of $S_{22}$ with time for $B=0.1$.

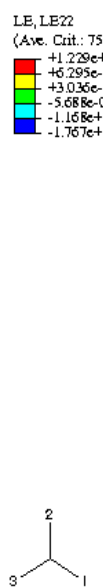

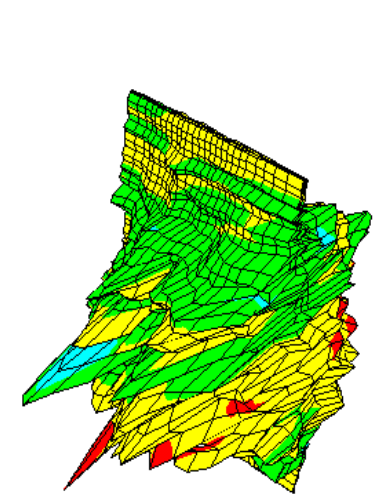

$h=1 \mathrm{~nm}$
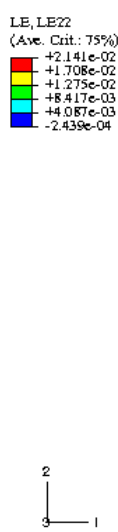

Figure 8. Strain contours showing the failure pattern for $B=0.0005$.

- Stress concentration failure or a crack propagation failure is defined as one in which the cohesive stress is reached only at those elements near the crack tip. The rest of the elements in the model are in a state of stress well below the cohesive stress value. Consequently, the subsequent loss of stiffness occurs only in a limited number of elements along which the crack propagation occurs.

Observations: For each $B$ value, it can be seen that as the thickness decreased from $10 \mathrm{~nm}$ to $1 \mathrm{~nm}$ the failure became a general failure. For thicker platelets, with thicknesses $4 \mathrm{~nm}$ and $10 \mathrm{~nm}$, the failure was due to stress concentration at the notch tip. The observations suggest that for thinner platelets the failure is general and not due to the presence of the notch, whereas in thicker platelets the presence of the notch 


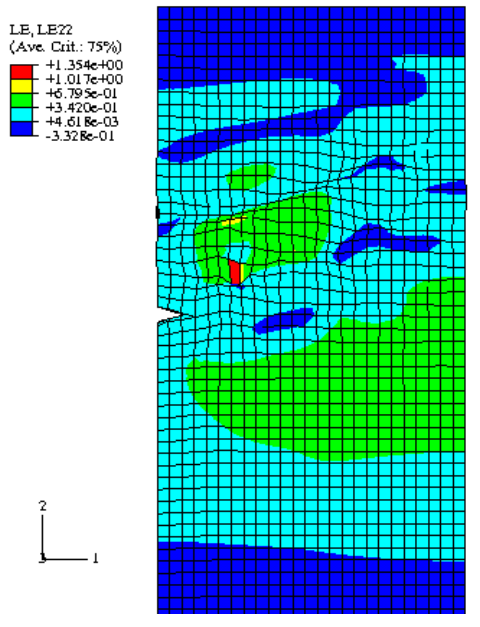

$h=1 \mathrm{~nm}$

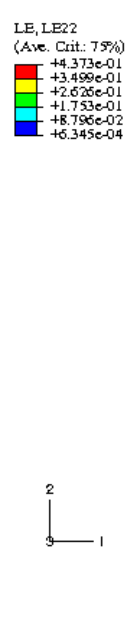

Figure 9. Strain contours showing the failure pattern for $B=0.005$.
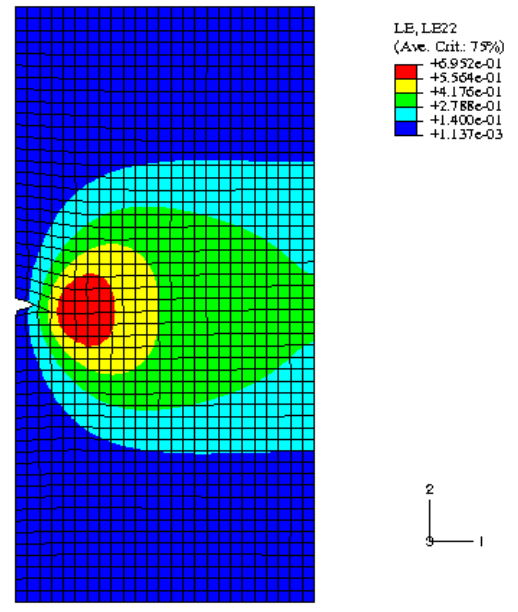

$h=4.25 \mathrm{~nm}$

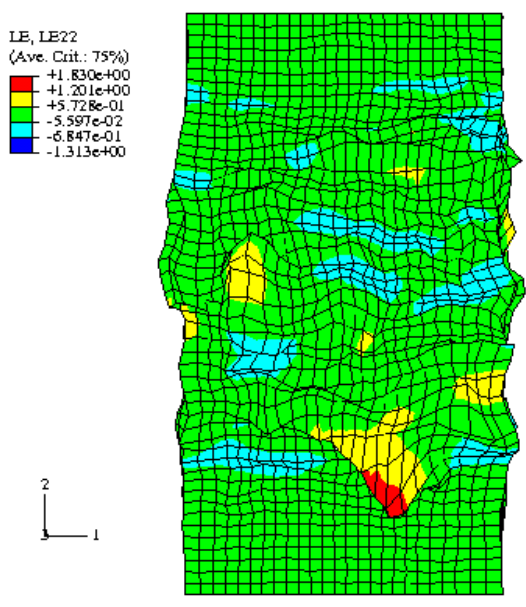

$h=1 \mathrm{~nm}$

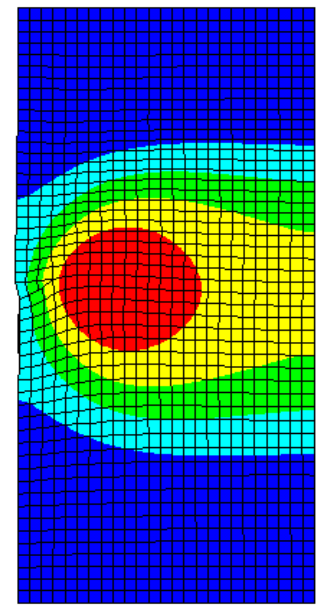

$h=10 \mathrm{~nm}$

Figure 10. Strain contours showing the failure pattern for $B=0.05$.

results in a stress concentration type failure of the platelet. The observed failure pattern suggests that, for a particular thickness, the change in the $B$ value does not change the manner in which the platelet fails. The behavior exhibited by the model results lend to the extrinsic length scale of the scaled VIB FE framework.

Stress-strain-time observations: This section presents select results of axial stress and strain and the times of failure for the different parameter values. These results are presented form in Table 1 on the next page. The following observations can be made from that table. 


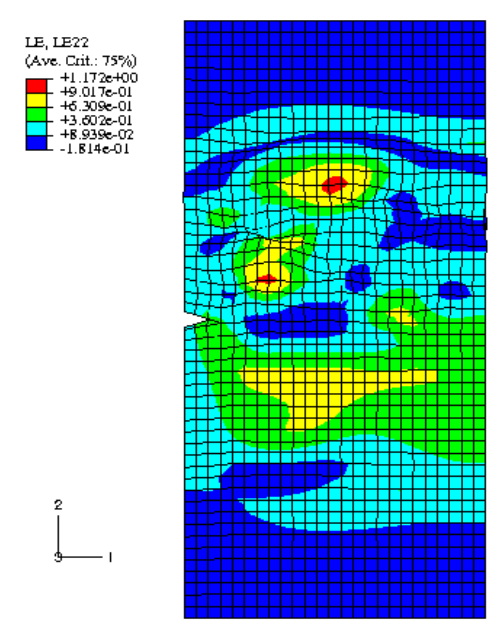

$h=1 \mathrm{~nm}$
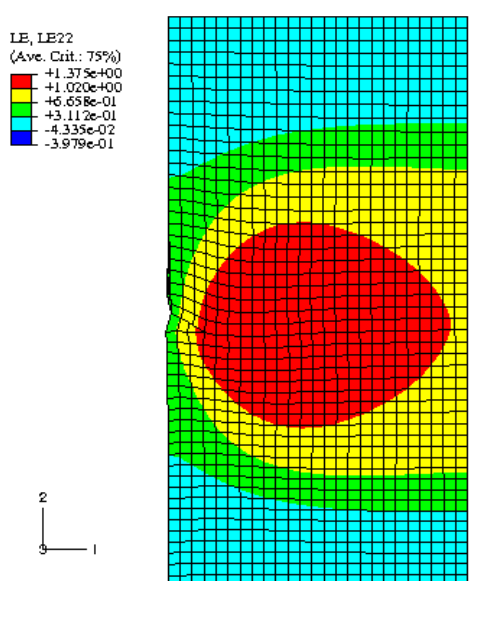

$h=4.25 \mathrm{~nm}$

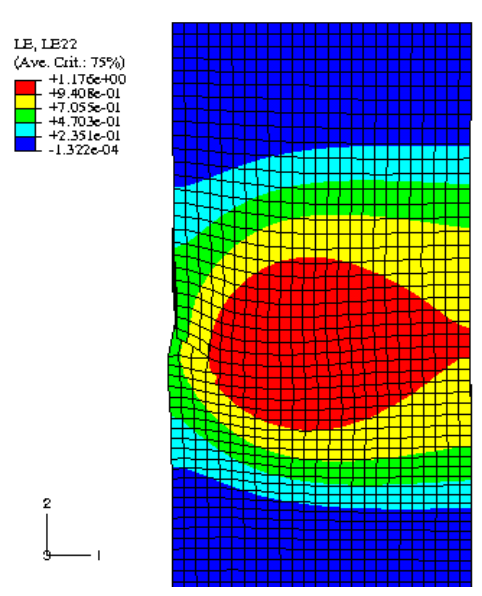

$h=10 \mathrm{~nm}$

Figure 11. Strain contours showing the failure pattern for $B=0.1$.

- Specific $B$ value: As thickness increases the stress and the time of failure increases. The increase in the time of failure indicates that the model is capable of taking a higher displacement at the ends before failure occurs.

- Specific thickness value: For a specific thickness as the $B$ value decreases the cohesive stress (failure stress) value decreases as expected.

- For all the thicknesses the failure stress and time for a $B$ value of 0.0005 remained the same, unlike for the other $B$ values. For $1 \mathrm{~nm}$ thickness the failure was of a general type while for thicknesses of $4 \mathrm{~nm}$ and $10 \mathrm{~nm}$ it was of a stress concentration type. This observation appears to indicate an

\begin{tabular}{|clccc|}
\hline Thickness & \multicolumn{1}{c}{$B$} & Time of failure & Stress at notch tip (MPa) & Strain at notch tip \\
\hline \multirow{2}{*}{$1 \mathrm{~nm}$} & 0.0005 & $1 \times 10^{-9}$ & 62.403 & 0.000412 \\
& 0.005 & $1 \times 10^{-9}$ & 104.16 & 0.000359 \\
& 0.05 & $1 \times 10^{-9}$ & 106.732 & 0.00035 \\
& 0.1 & $1 \times 10^{-9}$ & 105 & 0.00035 \\
\hline \multirow{2}{*}{$4 \mathrm{~nm}$} & 0.0005 & $1 \times 10^{-9}$ & 64.7658 & 0.000463 \\
& 0.005 & $3.5 \times 10^{-9}$ & 344.594 & 0.405 \\
& 0.05 & $3.5 \times 10^{-9}$ & 338 & 0.372 \\
& 0.1 & $3.5 \times 10^{-9}$ & 341 & 0.0014 \\
\hline \multirow{2}{*}{$10 \mathrm{~nm}$} & 0.0005 & $1 \times 10^{-9}$ & 65.1243 & 0.000472 \\
& 0.005 & $7.5 \times 10^{-9}$ & 594.624 & 0.0113 \\
& 0.05 & $7.5 \times 10^{-9}$ & 846.09 & 0.00513 \\
& 0.1 & $7.5 \times 10^{-9}$ & 854.97 & 0.0114 \\
\hline
\end{tabular}

Table 1. Stress-strain-time of failure with change in thickness. 
interesting suggestion that since the surface energy values for the material are not known a priori, a low $B$ value in the range of 0.0005 could be a reasonable value to be chosen for any further studies using minerals such as dahlite.

\section{Conclusions}

This paper has described a novel methodology, outlined, applied, and demonstrated issues involving scaled finite element virtual internal bond (VIB) modeling in an explicit finite element (FE) framework. The previous study [Thiagarajan et al. 2007] illustrated two FE implementation related characteristics, firstly that scaled properties can be used at the lower levels along with scaled dimensions and secondly that the loading time can be appropriately scaled without the loading becoming a dynamic loading. The scaled time aspect is significant as it allows for computations to be completed in real time which is about three to six orders faster than with traditional frameworks.

The parametric studies done and presented in this paper, using four different $B$ values and three different thicknesses for a total of twelve combinations, suggest that the $B$ values for minerals such as the one studied here should be in the range of 0.0005 .

The mesh size of a model plays an important role in the failure behavior of that particular model. The failure of any VIB model is represented by separation of the adjacent nodes near the crack tip which is caused due to localization of the strain in that region. Ji and Gao [2004] showed that for a material with $E=100 \mathrm{GPa}$ and $\gamma=1 \mathrm{~J} / \mathrm{m}^{2}$ with $B=0.06$ the localization zone is $3.5 \mathrm{~nm}$ wide. Since fracture simulation in the VIB model is often contained within one strip of the mesh, the mesh size has to be of the order of the localization zone. In the models that have been studied, the mesh size is of the order of the scale itself. Hence, in the cases of millimeter and micrometer models, as the mesh size is very large compared to the localization zone, the localization zone of nanometer length scale is seen in the model as a high stress zone near the notch tip which in turn results in a crack formation. In the nanometer scale model, since the mesh size is of the same order as the localization zone, the model shows a uniform distribution of the high stress resulting in the possibility that the model can fail anywhere, not necessarily at the notch tip. To have a nanometer mesh size in a millimeter or micrometer model will require prohibitively large computational times for this simulation.

\section{References}

[Akiva et al. 1998] U. Akiva, H. D. Wagner, and S. Weiner, "Modelling the three-dimensional elastic constants of parallel-fibred and lamellar bone”, J. Mater. Sci. 33:6 (1998), 1497-1509.

[Balooch et al. 2008] M. Balooch, S. Habelitz, J. H. Kinney, S. J. Marshall, and G. W. Marshall, "Mechanical properties of mineralized collagen fibrils as influenced by demineralization", J. Struct. Biol. 162:3 (2008), 404-410.

[Chang et al. 2005] D. Chang, G. Falini, S. Fermani, C. Abbott, and J. Moradian-Oldak, "Supramolecular assembly of amelogenin nanospheres into birefringent microribbons", Science 307:5714 (2005), 1450-1454.

[Currey 1990] J. D. Currey, "Physical characteristics affecting the tensile failure properties of compact bone", J. Biomech. 23:8 (1990), 837-844.

[Deshmukh 2006] K. P. Deshmukh, "Nano finite element modeling of the mechanical behavior of biominerals using a multiscale (virtual internal bond) model”, M.S. Thesis, University of Missouri, Kansas City, 2006.

[Eppell et al. 2001] S. J. Eppell, W. Tong, J. Katz, L. Kuhn, and M. J. Glimcher, "Shape and size of isolated bone mineralites measured using atomic force microscopy", J. Orthopaedic Res. 19:6 (2001), 1027-1034.

[Gao and Ji 2003] H. Gao and B. Ji, "Modeling fracture in nanomaterials via a virtual internal bond method", Eng. Fract. Mech. 70:14 (2003), 1777-1791. 
[Gao et al. 2003] H. Gao, B. Ji, I. Jager, E. Artz, and P. Fratzl, "Materials become insensitive to flaws at nanoscale: lessons from nature", Proceedings of the National Academy of Science 100:10 (2003), 5597-5600.

[Hulmes et al. 1995] D. J. Hulmes, T. J. Wess, D. J. Prockop, and P. Fratzl, "Radial packing, order, and disorder in collagen fibrils", Biophys. J. 68:5 (1995), 1661-1670.

[Jager and Fratzl 2000] I. Jager and P. Fratzl, "Mineralized collagen fibrils: a mechanical model with a staggered arrangement of mineral particles", Biophys. J. 79:4 (2000), 1737-1746.

[Ji and Gao 2004] B. Ji and H. Gao, "A study of fracture mechanisms in biological nano-composites via the virtual internal bond model”, Mater. Sci. Eng. A 366:1 (2004), 96-103.

[Katz 1976] J. L. Katz, "Hierarchical modeling of compact haversian bone as a fiber reinforced material", Adv. Bioeng., ASME (1976), 17-18. New York City.

[Katz 1980] J. L. Katz, “Anisotropy of Young's modulus of bone”, Nature 283:5742 (1980), 106-107.

[Kinney et al. 2003] J. H. Kinney, S. Habelitz, S. J. Marshall, and G. W. Marshall, "The importance of intrafibrillar mineralization of collagen on the mechanical properties of dentin", J. Dental Res. 82:12 (2003), 957-961.

[Landis et al. 1996] W. J. Landis, K. J. Hodgens, J. S. Min, J. Arena, S. Kiyonaga, M. Marko, C. Owen, and B. F. McEwen, "Mineralization of collagen may occur on fibril surfaces: evidence from conventional and high-voltage electron microscopy and three-dimensional imaging", J. Struct. Biol. 117:1 (1996), 24-35.

[Orgel et al. 2000] J. P. Orgel, T. J. Wess, and A. Miller, "The in situ conformation and axial location of the intermolecular cross-linked non-helical telopeptides of type I collagen", Structure 8:2 (2000), 137-142.

[Orgel et al. 2001] J. P. R. O. Orgel, A. Miller, T. C. Irving, R. F. Fischetti, A. P. Hammersley, and T. J. Wess, "The in situ supermolecular structure of type I collagen”, Structure 9:11 (2001), 1061-1069.

[Orgel et al. 2006] J. P. R. O. Orgel, T. C. Irving, A. Miller, and T. J. Weiss, "Microfibrillar structure of type I collagen in situ", Proc. Natl. Acad. Sci. USA 103:24 (2006), 9001-9005.

[Perumal et al. 2008] S. Perumal, O. Antipova, and J. P. R. O. Orgel, "Collagen fibril architecture, domain organization and triple-helical conformation govern its proteolysis”, Proceedings of the National Academy of Sciences 105:8 (2008), 28242829.

[Petruska and Hodge 1964] J. A. Petruska and A. J. Hodge, "A subunit model for the tropocollagen macromolecule", Proceedings of the National Academy of Sciences 51:5 (1964), 871-876.

[Rho et al. 1998] J. Y. Rho, L. Kuhn-Spearing, and P. Zioupos, "Mechanical properties and the hierarchical structure of bone", Med. Eng. Phys. 20:2 (1998), 92-102.

[Thiagarajan et al. 2004a] G. Thiagarajan, K. J. Hsia, and Y. Huang, "Finite element implementation of virtual internal bond model for simulating crack behavior", Eng. Fract. Mech. 71:3 (2004), 401-423.

[Thiagarajan et al. 2004b] G. Thiagarajan, Y. Y. Huang, and K. J. Hsia, "Fracture simulation using an elasto-viscoplastic virtual internal bond model with finite elements", J. Appl. Mech. (ASME) 71:6 (2004), 796-804.

[Thiagarajan et al. 2007] G. Thiagarajan, K. Deshmukh, Y. Wang, A. Misra, J. L. Katz, and P. Spencer, "Nano finite element modeling of the mechanical behavior of biocomposites using multi-scale (virtual internal bond) material models", J. Biomed. Mater. Res. A 83:2 (2007), 332-344.

[Tong et al. 2003] W. Tong, M. J. Glimcher, J. L. Katz, and S. J. Eppell, "Size and shape of mineralites in young bovine bone measured by atomic force microscopy", Calcified Tissue 72 (2003), 592-598.

[Veis 2005] A. Veis, "A window on biomineralization”, Science 307 (2005), 1419-1420.

[Weiner and Traub 1992] S. Weiner and W. Traub, "Bone structure: from angstroms to microns", FASEB J. 6 (1992), 879-885.

[Weiner and Wagner 1998] S. Weiner and H. D. Wagner, "The material bone: structure-mechanical function relations", Annu. Rev. Mater. Sci. 28 (1998), 271-298.

Received 7 Nov 2008. Revised 13 Aug 2009. Accepted 17 Aug 2009.

GANESH THIAGARAJAN: ganesht@umkc.edu

School of Computing and Engineering, University of Missouri, Kansas City, MO 64110, United States

KaVITA DeShMUKH: kavita81@gmail.com

School of Computing and Engineering, University of Missouri, Kansas City, MO 64110, United States 


\title{
JOURNAL OF MECHANICS OF MATERIALS AND STRUCTURES
}

\author{
http://www.jomms.org
}

\author{
Founded by Charles R. Steele and Marie-Louise Steele

\section{EDITORS} \\ Charles R. STEele \\ DAVIDE BIGONI \\ IWONA JASIUK \\ YASUHIDE SHINDO \\ Stanford University, U.S.A. \\ University of Trento, Italy \\ University of Illinois at Urbana-Champaign, U.S.A. \\ Tohoku University, Japan
}

\section{EDITORIAL BOARD}

H. D. BUI École Polytechnique, France

J. P. CARTER University of Sydney, Australia

R. M. Christensen Stanford University, U.S.A.

G. M. L. GLADWELL University of Waterloo, Canada

D. H. HodGES Georgia Institute of Technology, U.S.A.

J. HUTCHINSON Harvard University, U.S.A.

C. HwU National Cheng Kung University, R.O. China

B. L. KariHaloo University of Wales, U.K.

Y. Y. KIM Seoul National University, Republic of Korea

Z. Mroz Academy of Science, Poland

D. PAMPlonA Universidade Católica do Rio de Janeiro, Brazil

M. B. RUBIN Technion, Haifa, Israel

A. N. SHUPIKov Ukrainian Academy of Sciences, Ukraine

T. TARNAI University Budapest, Hungary

F. Y. M. WAN University of California, Irvine, U.S.A.

P. WRIGGERS Universität Hannover, Germany

W. YANG Tsinghua University, P.R. China

F. ZIEGLER Technische Universität Wien, Austria

\section{PRODUCTION}

\section{Paulo Ney de Souza Production Manager \\ SheIla Newbery Senior Production Editor \\ SILVIO LEVY Scientific Editor}

See inside back cover or http://www.jomms.org for submission guidelines.

JoMMS (ISSN 1559-3959) is published in 10 issues a year. The subscription price for 2010 is US $\$ 500 /$ year for the electronic version, and \$660/year (+\$60 shipping outside the US) for print and electronic. Subscriptions, requests for back issues, and changes of address should be sent to Mathematical Sciences Publishers, Department of Mathematics, University of California, Berkeley, CA 94720-3840.

JoMMS peer-review and production is managed by EditFLOW ${ }^{\mathrm{TM}}$ from Mathematical Sciences Publishers.

PUBLISHED BY

mathematical sciences publishers

http://www.mathscipub.org

A NON-PROFIT CORPORATION

Typeset in LATEX

CCopyright 2010. Journal of Mechanics of Materials and Structures. All rights reserved. 


\title{
Journal of Mechanics of Materials and Structures
}

\author{
Volume 5, No. 2 February 2010
}

A critical analysis of interface constitutive models for the simulation of delamination in composites and failure of adhesive bonds

Anton Matzenmiller, Sebastian Gerlach and Mark Fiolka

185

Computational studies of collagen fibril biominerals using a virtual internal bond

model with extrinsic length scale

Ganesh Thiagarajan and Kavita Deshmukh

The simulation of stochastically excited viscoelastic systems and their stability

VADIM D. POTAPOV

Fundamental solutions for an inhomogeneous cross-anisotropic material due to horizontal and vertical plane strain line loads

Cheng-Der Wang, Jia-YAN Hou and WeI-Jer WANG

Mechanical and fracture analysis of welded pearlitic rail steels

Aldinton Allie, Heshmat A. Aglan and Mahmood Fateh

Rate dependence of indentation size effects in filled silicone rubber

Ramanjaneyulu V. S. TATIRAJU and ChUng-SOUK HAN

A novel application of a laser Doppler vibrometer in a hèalth monitoring system

DAVOOD REZAEI and FARID TAHERI

Energy absorption of a helicoidal bistable structure

Seubpong Leelavanichkul, Andrej Cherkaev, Daniel O. Adams

and FLORIAN SOLZBACHER

Decay properties of solutions of a Mindlin-type plate model for rhombic systems

Francesca Passarella, Vincenzo Tibullo and VitTorio Zampoli

A consistent refinement of first-order shear deformation theory for laminated composite and sandwich plates using improved zigzag kinematics Alexander Tessler, Marco Di Sciuva and Marco Gherlone 